\title{
The circles of light
}

\author{
Synchrotron radiation has revolutionized our ability to probe and understand the intrinsic \\ properties of materials. A new generation of synchrotron facilities and techniques is emerging, \\ extending our capabilities in materials characterization.
}

One of Superman's many abilities is his X-ray vision, which enables him to see through objects. Indeed, the discovery of X-rays in the late 19th century and the realization that these could be used to 'see' inside the human body, and later to determine the structure of crystals, must have then seemed superhuman. Yet today, light from across the electromagnetic spectrum is routinely used to image and probe diverse systems. The development of synchrotrons and the discovery of synchrotron radiation - ultra-bright light emitted when charged particles are accelerated to high speeds along a circular path - gave researchers the tools to selectively explore and extract information from a wide range of materials. Synchrotron techniques are now central to many fields and are being used to investigate critical issues, such as the energy-storage problem and antibiotic resistance; to unlock the secrets of historical artefacts; and to record molecular movies.

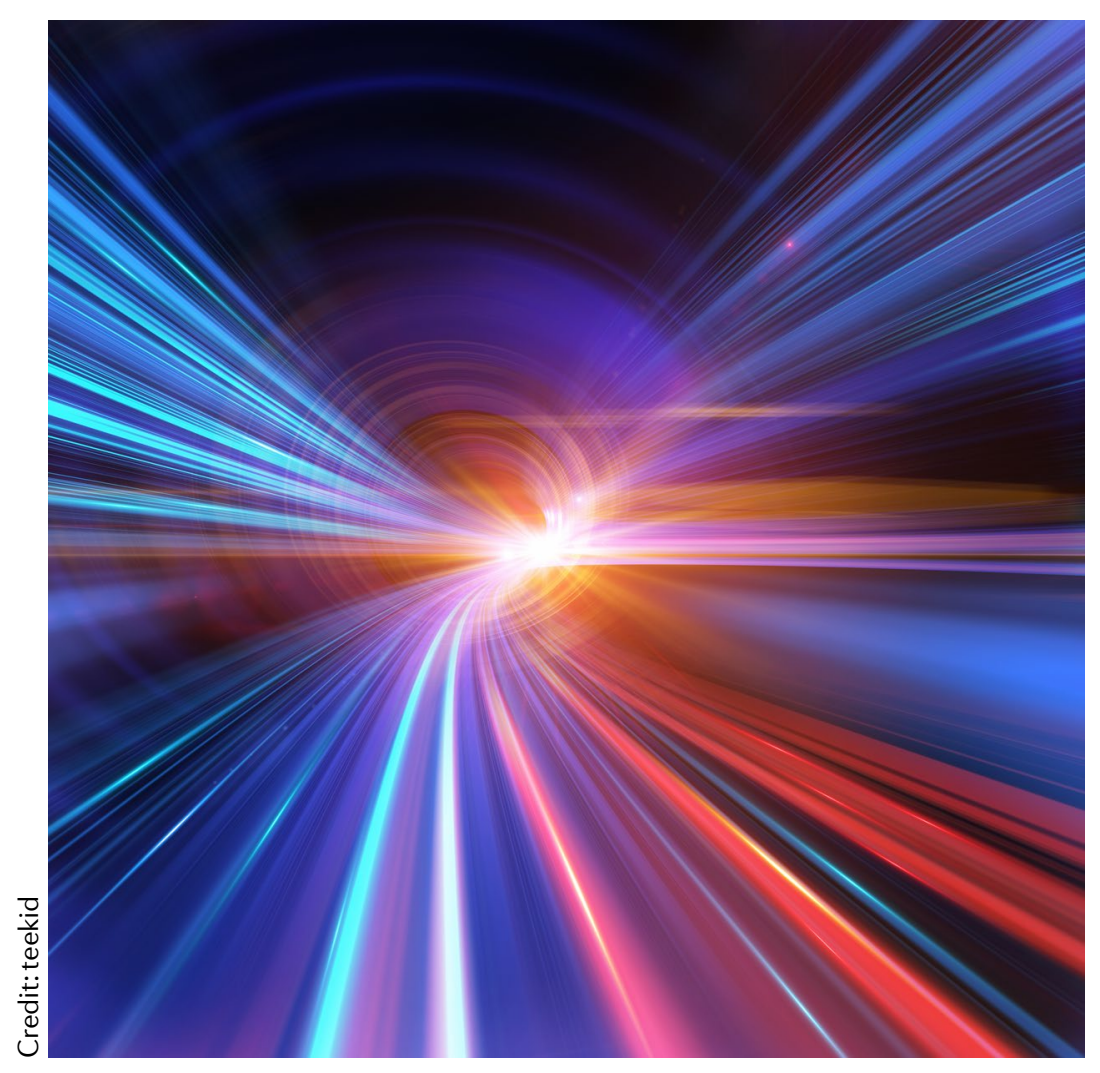

Today's facilities have come a long way from the firstgeneration synchrotrons of the 1950s onwards that were designed for the study of high-energy particle physics, with synchrotron radiation as a by-product. In 1980, the United Kingdom built the world's first synchrotron specifically designed to generate ultra-bright radiation for research. We are now at the cusp of a new era fourth-generation synchrotrons are under construction and X-ray free-electron lasers (XFELs) are emerging as powerful and highly tunable $\mathrm{X}$-ray sources. These new facilities and instruments are extending the boundaries of what we can achieve, offering increased brilliance and shorter pulse lengths, which will enable dynamic properties of materials to be measured on fundamental length- and timescales. A sample of the possible techniques and applications is showcased in this Focus issue on synchrotron techniques.

One of the aims of condensed-matter physics is to manipulate and control the properties of quantum materials for the realization of novel electronic and photonic technologies. Using synchrotron light is one of the principal approaches to investigate this diverse class of materials, which are typically strongly correlated and have exotic properties that are highly dependent on the complex interplay between numerous internal degrees of freedom. In their Review, Yulin Chen and co-authors examine how angle-resolved photoemission spectroscopy is being used to establish relationships between the electronic structure of quantum materials and their macroscopic properties - a key step on the path to technological application. Another Review, penned by Andrea Cavalleri and colleagues, describes how the advent of femtosecond X-rays has increased our understanding and enabled control of non-equilibrium phenomena in quantum materials. The challenge of studying non-equilibrium phenomena in highly correlated systems is not restricted to experimental studies, with Thomas Devereaux and co-authors reviewing the state-of-the-art theoretical approaches being used to evaluate equilibrium and non-equilibrium spectroscopic techniques.

Establishing the fundamental relationship between the structure and performance of materials remains a goal of many fields, in particular heterogeneous catalysis, for which understanding dynamic changes during operation is of importance. The advancement of in situ and operando synchrotron techniques is beginning to enable 
us to observe changes in systems such as lithium-ion batteries and solid-state catalysts in real time under working conditions. In their Review, Bert Weckhuysen and Florian Meirer provide a historical overview and assess the latest developments in the application of X-ray and infrared synchrotron techniques to heterogeneous catalysts. Additionally, in a Comment, Vanessa Wood dicusses how X-ray tomography is contributing to the development of lithium-ion batteries.

Undoubtedly, there have been impressive developments in synchrotron techniques, but there remain limitations, such as limits to the spatial and temporal resolution. To this end, commissioning fourth-generation synchrotrons with improved performance represents a major step towards overcoming these limitations. Europe is home to two of the most advanced facilities: MAX IV (Sweden) is the first fourth-generation synchrotron to come online and the European XFEL (Germany) is the first of a new generation of XFELs with an ultrahigh repetition rate. Facilities are also under construction in other parts of the world, with the next generation of XFELs including an upgrade of the Linac Coherent Light Source (LCLS, USA) and the construction of the Shanghai Coherent Light Facility (China). In a Comment, Mike Dunne, Director of the LCLS, discusses the technological developments underpinning next-generation XFELs and the research directions in materials science that these will enable.

The construction of synchrotron facilities in new geographical locations opens the door to many more users, offering possibilities in terms of research and collaboration. For example, the opening of SESAME (Synchrotron-light for Experimental Science and Applications in the Middle East) in Jordan last year was a milestone for the region. In their Comment, Rolf Heuer, President of the SESAME Council, and James
Gillies describe the progress made in SESAME's first year of operation. SESAME is also a prime example of the importance of international collaboration in the development and operation of major facilities, a sentiment echoed in the Feature article on the future of X-ray and neutron beamlines in Europe by Christine-Maria Horejs, an editor on the Nature Reviews Materials team.

Public engagement and educational outreach are increasingly important considerations for national and international neutron spallation and synchrotron facilities. Cultural heritage applications, such as the preservation of precious artefacts for future generations, showcase the benefits of such facilities. Writing in a Comment, Eleanor Schofield, Head of Conservation and Collections Care at the Mary Rose Trust, explains how synchrotron X-ray analysis is contributing to the preservation of Henry VIII's warship, the Mary Rose. In our first In the Classroom article, Joseph Bevitt, from the Australian Nuclear Science and Technology Organisation, describes the benefits of involving local children in the analysis of data generated from X-ray and neutron studies of dinosaur fossils. Indeed, all over the world, synchrotron facilities are inspiring the next generation of synchrotron scientists, with examples of educational projects including the successful completion of Project $\mathrm{M}$ at the Diamond Light Source (UK) and the recent launch of Misión ALBA (Spain) with the motto "making visible the invisible". It is this same desire that must have driven the scientists of the late 19 th and early 20 th centuries who pioneered the use of X-rays to probe matter and continues to inspire scientists today.

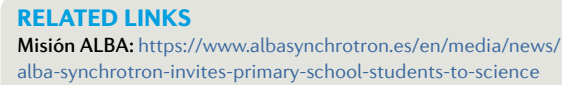

